\title{
Magnetic Field Measurements of The Harmonic Generation FEL Superconducting Undulator at BNL-NSLS
}

\author{
L. Solomon and W.S.Graves \\ National Synchrotron Light Source, Brookhaven National Laboratory, Upton, N.Y. 11973, USA
}

\section{I.Lehrman}

Grumman Aerospace Corporation, 4 Independence Way, Princeton, N.J., USA.

A three stage superconducting undulator (modulator, dispersive section, and radiator) is under construction at Brookhaven National Laboratory. Sections of the radiator, consisting of $25 \mathrm{~cm}$ long steel yokes, each with $18 \mathrm{~mm}$ period, 0.54 Tes 1 . field, and $8.6 \mathrm{~mm}$ gap are under test. The magnetic measurements and operationai characteristics of the magnet are discussed. Measurement results and analysis are presented, with emphasis on the integrated field quality. The magnet winding and the effects of the various trims are discussed.

This work has been performed under the auspices of the U.S.Department of Energy

\section{DISCLAIMER}

This report was prepared as an account of work sponsored by an agency of the United States Government. Neither the United States Government nor any agency thereof, nor any of their employees, makes any warranty, express or implied, or assumes any legal liability or responsibility for the accuracy, completeness, or usefulness of any information, apparatus, product, or process disclosed, or represents that its use would not infringe privately owned rights. Reference herein to any specific commercial product, process, or service by trade name, trademark, manufacturer, or otherwise does not necessarily constitute or imply its endorsement, recommendation, or favoring by the United States Government or any agency thereof. The views and opinions of authors expressed herein do not necessarily state or reflect those of the United States Government or any agency thereof. 


\section{INTRODUCTION}

A three stage superconducting undulator for use in a high gain, harmonic generation experiment is under construction at the National Synchrotron Light Source at Brookhaven National Lab. [1-4] The device triples the frequency of a 10.4 micron $\mathrm{CO}_{2}$ seed laser using a $30 \mathrm{MeV}$ electron beam. The first stage of the undulator, the modulator section (12 periods, $26 \mathrm{~mm}, 0.81 \mathrm{~T}$ ), modulates the energy of the electron beam. The second stage, the dispersive section $(12 \mathrm{~cm}, 0.3-1.2 \mathrm{~T})$, spatially bunches the energy modulated beam. The final stage, the radiator section ( 84 periods, $18 \mathrm{~mm}, 0.54 \mathrm{~T}$, tapering possible), extracts energy from the coherently bunched beam at the third harmonic of the modulator.

The magnet yokes for all three stages of the undulator are machined out of ground low carbon steel blocks, and are fabricated by Grumman Corporation. The radiator stage is made up of six contiguous $25 \mathrm{~cm}$ long sections, each section consisting of an upper and lower magnet yoke. A series of slots is precision machined $( \pm 10 \mu \mathrm{m})$ into the yokes, and the superconducting wires ( $\mathrm{SSC}$ wire- $\mathrm{Cu} / \mathrm{SC}=2.4: 1,0.465 \mathrm{~mm} \mathrm{OD}$ with Kapton wrapping) are wound within these slots. Machining errors result in $0.1 \%$ peak field errors in the unsaturated regime $\left(\frac{\delta B}{B} \approx \frac{\delta g}{g}\right)$, without trimming or shimming [3]. The process of clamping and aligning the individual magnet yokes into sections and groups of sections must preserve these tight tolerances, requiring careful assembly and mechanical measurement. The main magnet winding is continuous, with each slot being wound in the reverse direction from its neighboring slots.

Among the attributes of this superconducting undulator technology [5] are high fields at small periods and gaps, an easily varied field and field taper, and magnet entrance and exit conditions modifiable through changes in the wire winding or the end trim currents. The magnets reported on in this paper have all been wound with a binomial current pattern [6] which results in a net displacement but no steering, in the absence of any saturation effects.

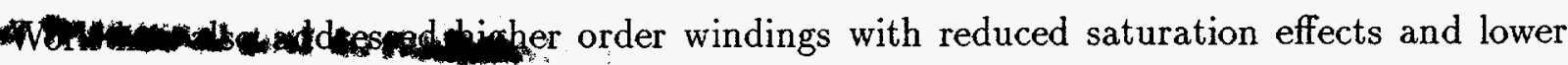
steering end correction requirements [7].

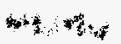




\section{DISCLAIMER}

Portions of this document may be illegible in electronic image products. Images are produced from the best available original document. 


\section{MAGNET WINDING}

The winding scheme of the radiator magnet has been chosen to permit maximum flexibility in powering of the yoke windings without unnecessary complexity. The yokes are wound identically so that sorting of the magnet yokes is possible. Each radiator yoke has 28 slots, and five independent coils wound on it - the main winding and two entrance and two exit windings. The main winding is a single continuous winding, and is wound as a binomial pattern with 2 wire layers in slot 1 and 28,6 layers in 2 and 27 , and 8 layers in slots 3 through 26. The entrance and exit trims each consist of a single slot trim ( 4 layers in slot 1 , and 4 layers in slot 28) and a double slot trim ( 2 layers in slot 2 and 3, and 2 layers in slot 27 and 28). Thus, all slots have 8 layers of windings. The six radiator sections are assembled contiguously to form the full length radiator. All the windings are connected in series except for the entrance windings on section 1, and the exit windings on section 6 . However, the connection points between the various windings are connected to power leads, so that differential powering of the various trims is possible. In this way, the entrance and exit trims on the interior magnet sections of the radiator can function as both joint trims and taper windings. With this scheme, the taper transitions are also binomial. Characterization of the entrance and exit trim windings, the windings in the vicinity of the magnet joints, and the taper transitions is in progress. As the saturation level of the magnet increases, the nominally steering free current distribution pattern at the magnet entrance and exit deviates from the ideal, and the beam is steered off axis. The entrance and exit trim windings are able to correct this steering effect, but higher order windings which reduce the saturation dependence and the required end trim currents are being tested [7].

\section{TESTING AND RESULTS}

The radiator magnets are tested in $50 \mathrm{~cm}$ long units, i.e. 2 sections long (4 magnet yokes). A slider supporting a Hall probe array is guided by the gap spacer around which the magnet 
yokes are clamped. A helmholtz coil in the test setup permits in-situ calibration of the Hall probes at helium temperatures. Details and errors associated with the measurement system are described elsewhere [3]. The nominal operational current for the radiator magnet is 90 amps, where the magnet is in a saturated state [5]. The quench current for these magnets, which do not exhibit any training behavior, is $\approx 180$ amps.

As with any iron magnet system, there is concern about hysteris affecting the reproducibility of a state of the magnet. With the radiator yokes, reliable setpoints are obtained if the magnet is cycled to $+/-175 \mathrm{amps}$, with subsequent setting of the operating current on the approach towards zero current. In order to assess both the reliability of the setting of a given magnet state and the repeatability of the measurement system, a data set consisting of several field scans at a setpoint of 90 amps was taken. The magnet was then cycled several times to $+/-175 \mathrm{amps}$, the magnet current was reset to $90 \mathrm{amps}$, and another data set of several field scars were taken. There were essentially no differences between tin intra-set data and the inter-set data in terms of field integral and RMS integral variations (10G-cm). However, after a current setpoint is reached, the magnetic field approaches and equilibrium value fairly slowly, as seen in Figure 1. Though the magnitude of the variation is relatively small, a fit of the data to the form $\exp -(t / \tau)$ yields $\tau=8$ minutes, shown as the solid line in Figure 1. To accommodate this time behavior the magnet is stabilized before data acquisition.

Measurements have been taken both with and without field clamps at either end of the test sections, with a dipole loop wrapped around the yokes, and with the end trims powered to straighten the electron beam trajectory. Shown in Figure 2 is a plot of the electron beam trajectory calculated from twice integrated field data taken at $90 \mathrm{Amps}$, with the entrance and exit trims powered symmetrically, and a dipole loop powered to provide $\approx 12$ Gauss of field. In this data all the interior poles have the same current excitation; none of the coils in the joint area have been powered differentially. Excluding the first and last pole, the total movement of the electron beam centroid is about $+/-25 \mu \mathrm{m}$ peak-to-peak, which is well within the required limit of an RMS variation of $+/-20 \mu \mathrm{m}$ (1/10th of the beam radius). 
Consistent with earlier data, the peak field variation is $0.27 \%$ RMS.

Given this high quality trajectory through two sections of the radiator, it is clear that the internal magnet errors are clearly low enough that correctors within the body of the magnet are not required. However, the entrance/exit region, and the joint region requires further investigation. If the end trims can systematically straighten the entrance/exit trajectory, the entire six radiator sections can be assembled with confidence in the resulting trajectory quality. To this end, a parametric study of the effects of the various trims (the double slot trim, the single slot trim, and the joint trim) was carried out. The results of varying the double slot trim (main winding at 90 amps, with field clamps) are shown in Figure 3. The upper two plots are scans at two different excitations of the double slot trim (21 and 28 amps). In order to test how closely the double slot trim approximates a point kick at the entrance of the magnet, a simulated kick was introduced into the data (i.e. a slope is superimposed onto the second integral results) "he lowest plot in Figure 3 shows the result of this manipulation, displaced vertically for clarity. It is clear that the end trim functions as a variable entrance/exit kick, with the deviation from this approximation being localized to the region near the ends of the magnet. In this sense, the trim acts locally to kick the electron beam. The same result is seen in studies of the single slot entrance/exit trim.

The physical joint between two radiator sections is an area of concern due to the possible errors and misalignments which might occur. As mentioned previously, the windings in the region of a physical joint can be powered as joint correction trims. That is, in addition to the main current in the winding, a small current can be superimposed onto the joint area windings to augment or oppose the main current. On one test assembly there was an inadvertent joint field error of $\approx 2 \%$ which resulted in a trajectory error shown Figure 4 , upper trajectory. Though the source of this particular error has not yet been identified, attempts to correct the error through powering the joint windings were highly successful. Shown as the middle and lower trajectories (displaced vertically for clarity) are the results of two attempts to modify the joint region through the joint winding trims. Clearly, the middle plot compensates the joint error quite well, while the lower scan overcompensates. 
Though precautions will be taken to insure that joint errors are minimized, the existing windings on the yokes can correct the resultant error integrals if they do occur.

Further work includes measurements of the radiator sections with a single period transverse and vertical field coil, additional measurements on the field profile due to the parabolic pole faces, and characterization of the modulator and dispersive stages. The cryostat and full length undulator clamping and alignment assembly is being fabricated by Grumman Co. When all the undulator stages are installed in the cryostat the total assembly will be measured. A measuring fixture will move inside the $8 \mathrm{~mm}$ diameter vacuum chamber which uses both optical and magnetic field measurements to determine the field profile of the device. With these measurements, current settings of the various magnet windings will be determined for the operating points of the undulator.

The authors would like to acknowledge discussions with, and contributions of I. Ben-Zvi, K. Halbach, S. Krinsky, G. Rakowsky, and L.H.Yu through the ' surse of the work presented here. 


\section{REFERENCES}

[1] L.H.Yu Phys. Rev A 99 (1991)5178

[2] I.Ben-Zvi et.al.NIM A 318 (1992)208

[3] L.Solomon, G.Ingold, I.Ben-Zvi, S.Krinsky, L.H. Yu, W.Sampson, K.Robins Proceedings of the 1993 Particle Accelerator Conference (1993) 1602

[4] G.Ingold, L.Solomon, I.Ben-Zvi, S.Krinsky, D.Li, D.Lynch, J.Sheehan, M.Woodle, X.Z.Qiu, L.H.Yu, X.Zhang, W.Sampson, M.Garber, K.Robins, I.Lehrman, R.Heuer, J.Sheehan, and D.Weissenburger Proceedings of the 1993 Particle Accelerator Conference (1993) 1439

[5] G.Ingold, L.Solomon, I.Ben-Zvi, S.Krinsky, D.Li, D.Lynch, J.Sheehan, M.Woodle, X.Z.Qiu, L.H.Yu, X.Zhang, W.Sampson, M.Garber, K.Robins, I.Lehrman, R.Heuer, J.Sheehan, and D.Weissenburger SPIE 1993 Symposium on Electron-Beam Sources of High Brightness Radia tion San Diego, SPIE Proceedings Vol. 2013 (1993) 68

[6] K.Halbach Nuclear Instruments and Methods A250 (1986) 95

[7] W.S.Graves, L.Solomon, and I.Lehrman Proceedings of the 1994 FEL Conference 


\section{FIGURES}

FIG. 1. The time behavior of the magnetic field measured after a change in the current setpoint.

FIG. 2. The electron trajectory through two radiator sections at the nominal operating current of 90 Amps.

FIG. 3. The effect of the double slot trim on the electron trajectory - (top) - Double Slot Trim at 21 amps; (Middle) - Double Slot Trim at 28 amps; (Bottom) - 21 amps data with a simulated point kick, displaced vertically for clarity

FIG. 4. The electron trajectory through two radiator sections at three different current settings of the joint trims, displaced vertically for clarity 


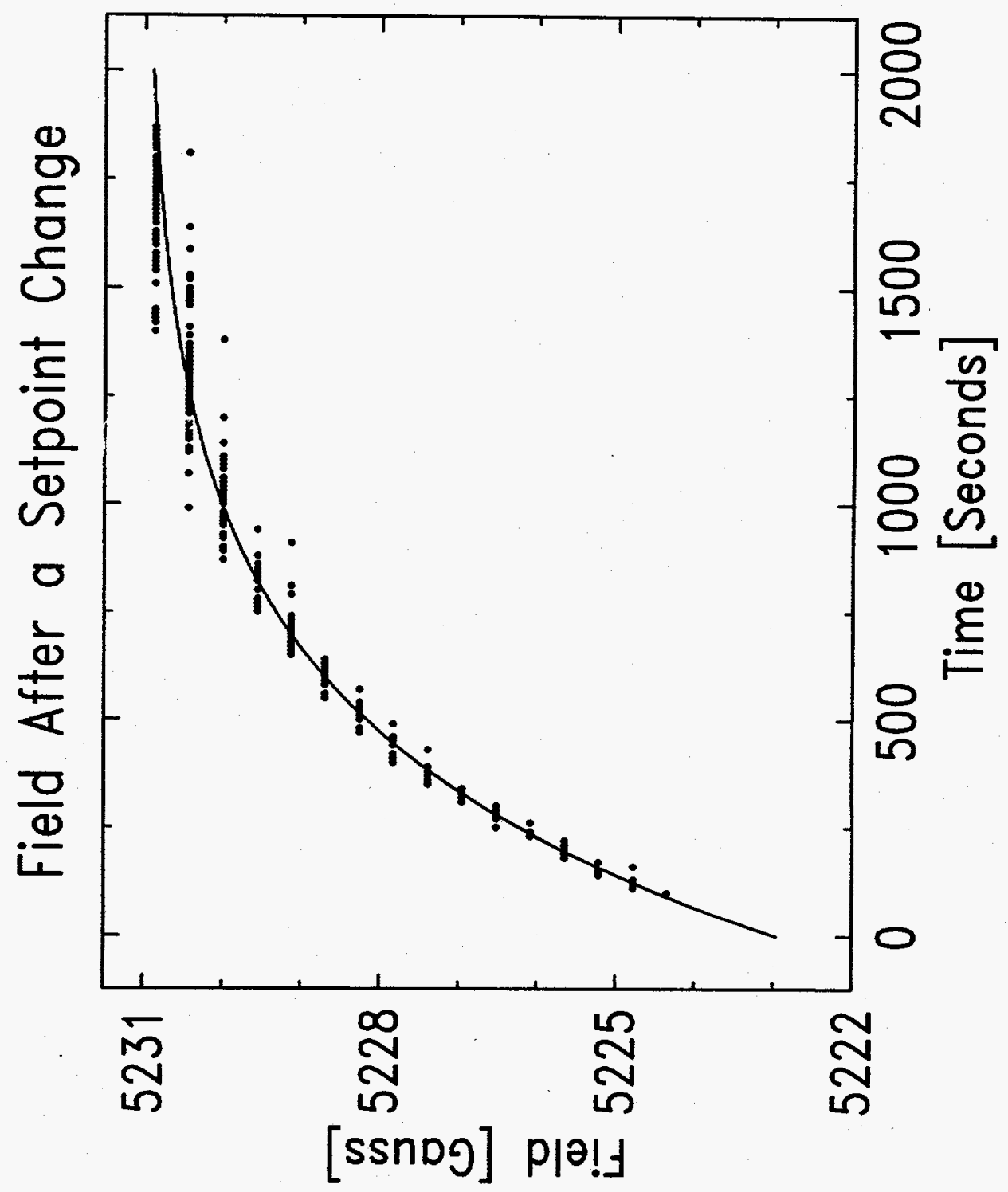

$F \mid G 1$ 


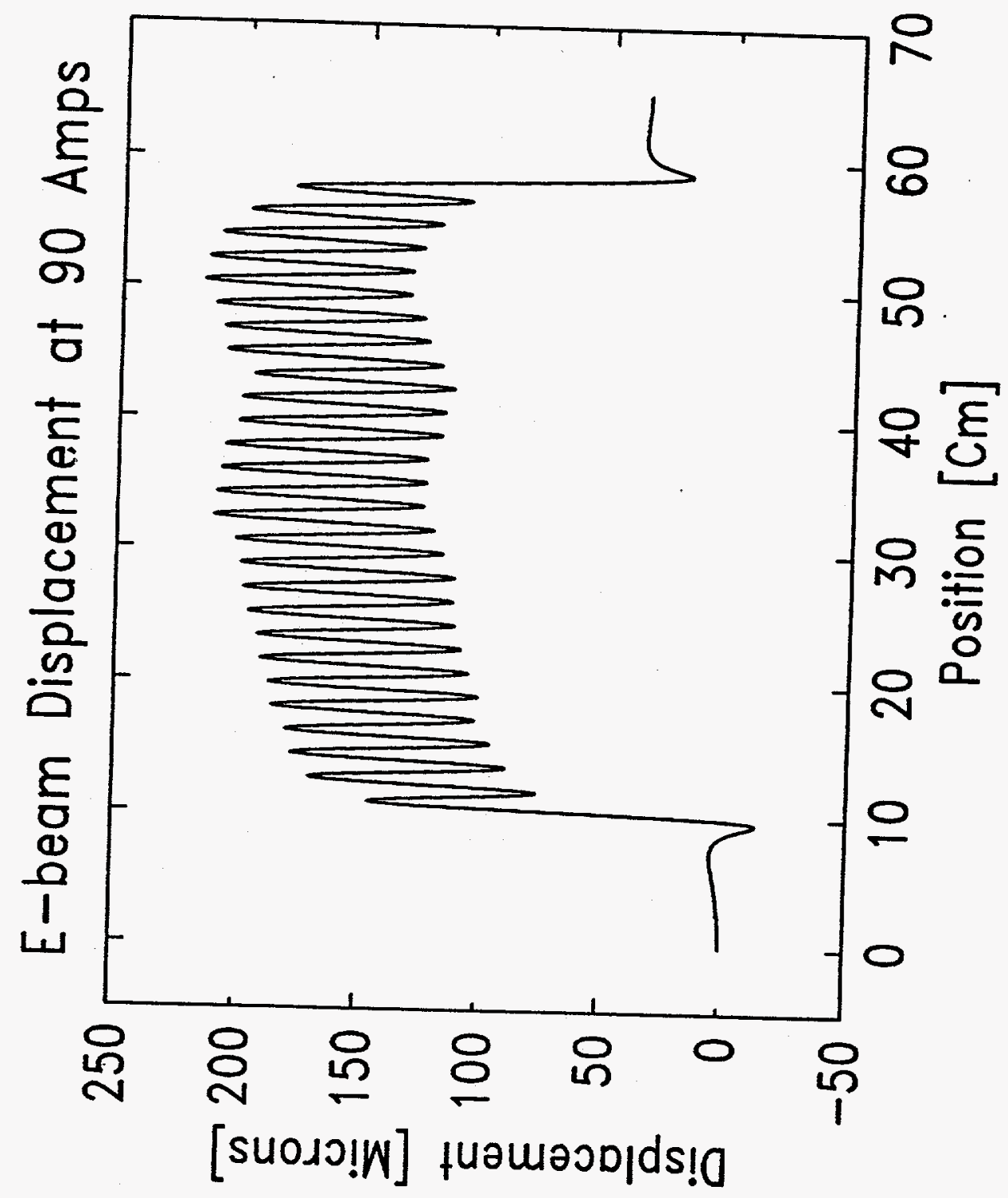

FIG 2 


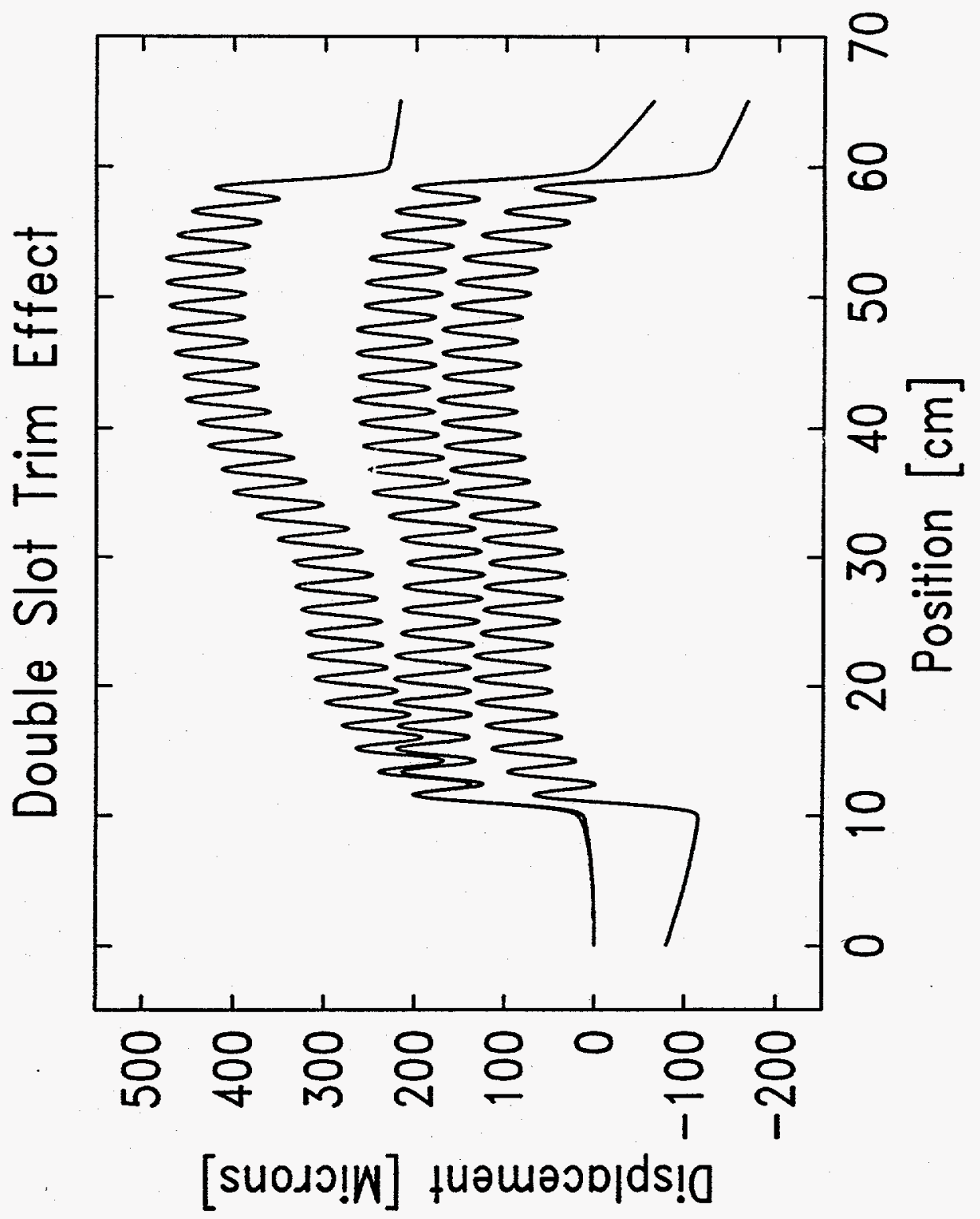

FIG 3 


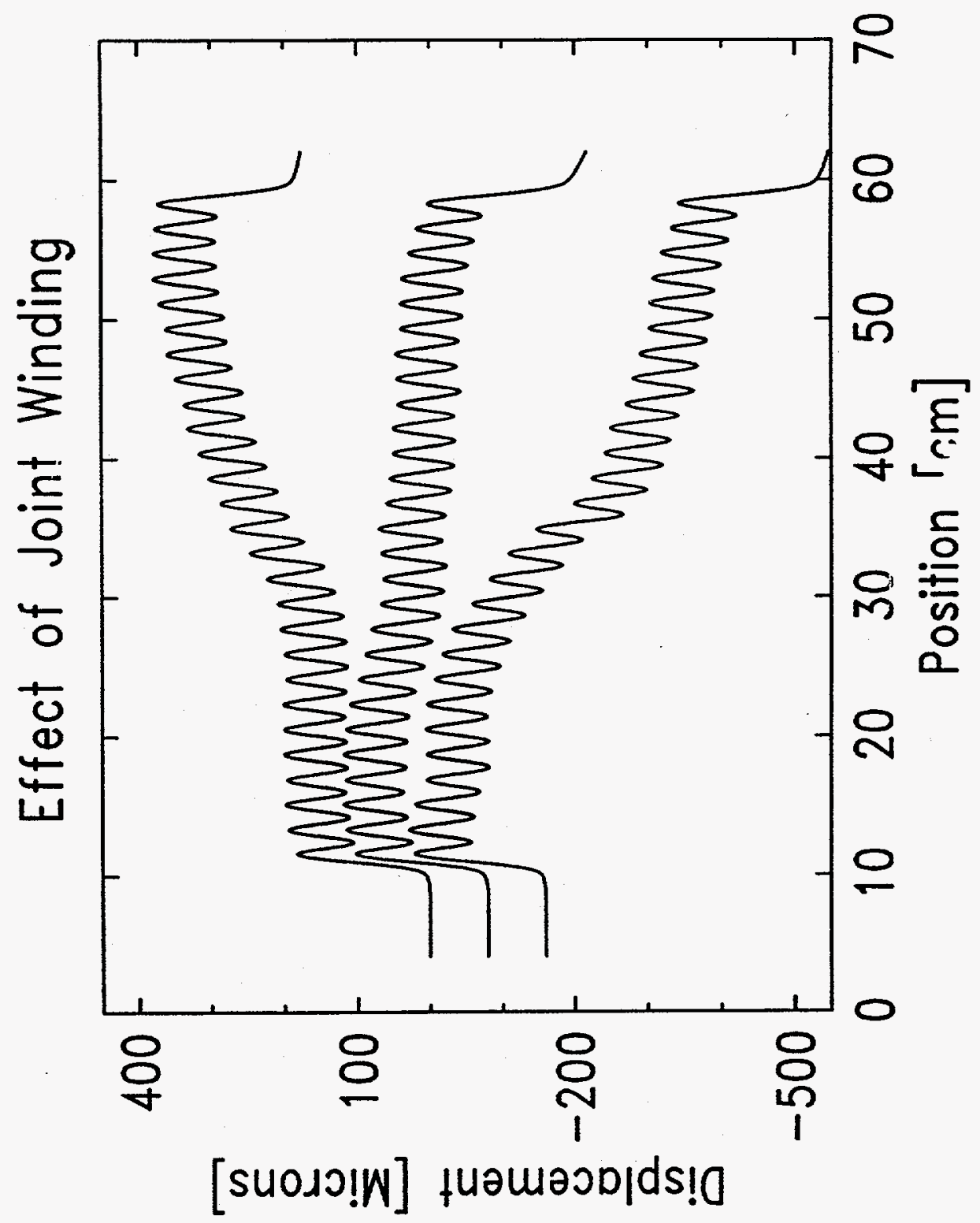

FIGURE 4 